\title{
Germline organization in Strongyloides nematodes reveals alternative differentiation and regulation mechanisms
}

\author{
Arpita Kulkarni $^{1}$ - James W. Lightfoot ${ }^{1}$ • Adrian Streit ${ }^{1}$
}

Received: 17 August 2015 /Revised: 15 November 2015 / Accepted: 16 November 2015/Published online: 12 December 2015

(C) The Author(s) 2016. This article is published with open access at Springerlink.com

\begin{abstract}
Nematodes of the genus Strongyloides are important parasites of vertebrates including man. Currently, little is known about their germline organization or reproductive biology and how this influences their parasitic life strategies. Here, we analyze the structure of the germline in several Strongyloides and closely related species and uncover striking differences in the development, germline organization, and fluid dynamics compared to the model organism Caenorhabditis elegans. With a focus on Strongyloides ratti, we reveal that the proliferation of germ cells is restricted to early and mid-larval development, thus limiting the number of progeny. In order to understand key germline events (specifically germ cell progression and the transcriptional status of the germline), we monitored conserved histone modifications, in particular H3Pser10 and $\mathrm{H} 3 \mathrm{~K} 4 \mathrm{me}$. The evolutionary significance of these events is subsequently highlighted through comparisons with six other nematode species, revealing underlying complexities and variations in the development of the germline among nematodes.
\end{abstract}

James W. Lightfoot and Adrian Streit contributed equally as principle investigators.

Electronic supplementary material The online version of this article (doi:10.1007/s00412-015-0562-5) contains supplementary material, which is available to authorized users.

Adrian Streit

adrian.streit@tuebingen.mpg.de

1 Department Evolutionary Biology, Max Planck Institute for Developmental Biology, D-72076 Tübingen, Germany
Keywords Germline $\cdot$ Nematodes $\cdot$ Strongyloides $\cdot$ Histone modification $\cdot$ Germline chromatin

\section{Introduction}

Roundworms of the genus Strongyloides are widespread small intestinal parasites of various vertebrates (Viney and Lok 2015). Several members of this genus are being developed as model organisms and are particularly useful for parasitological research (of medical and veterinary interest) and for the study of basic biological questions such as host parasite interactions (Bleay et al. 2007; Crook and Viney 2005; Viney et al. 2006) and evolution (Fenton et al. 2004; Gemmill et al. 2000; Streit 2014). Additionally, the genus Strongyloides is in an interesting position phylogenetically, having close relatives representing extremely divergent modes of reproduction and lifestyles, ranging from being free-living (further subclassified as facultative or obligate animal parasites) to entomopathogenic and even plant parasitic (Fig. 1a) (Blaxter et al. 1998; Holterman et al. 2006). Therefore, Strongyloides spp. and their relatives have great potential for further development as highly informative models for comparative evolutionary studies. Our current understanding of such evolutionary aspects comes from other nematode species like the well-established (but phylogenetically distantly related) nematode models Caenorhabditis and Pristionchus spp. (Sommer and Bumbarger 2012). The rat parasite Strongyloides ratti (S. ratti) and the sheep parasite Strongyloides papillosus (S. papillosus) have been developed as model representatives of this genus (Eberhardt et al. 2007; Viney 1999; Viney and Lok 2015). While $S$. ratti can be conveniently maintained in their natural host, $S$. papillosus is maintained in rabbits, which act as permissive laboratory hosts. The possibility of and ease in accessing free-living stages, in addition to a number of 
A

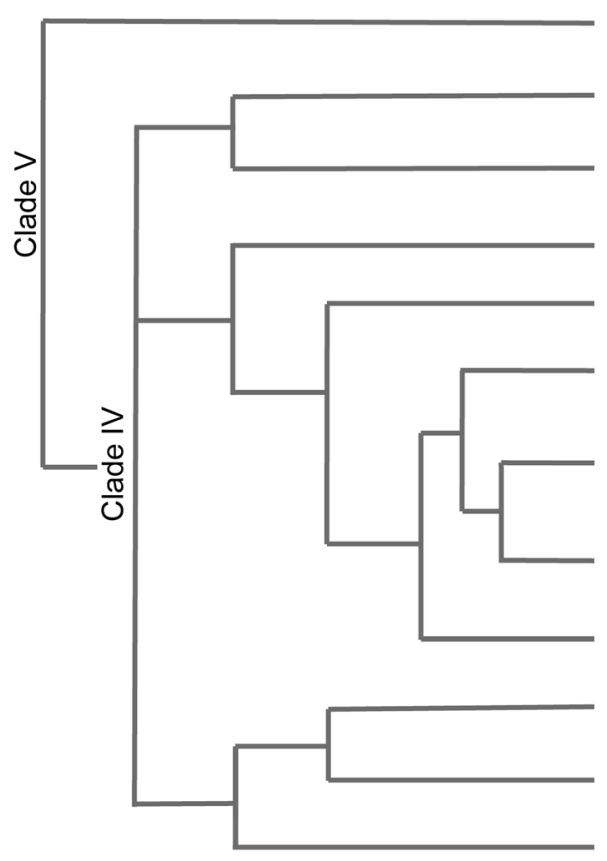

C. elegans (Free-living; self-fertile hermaphrodites \& males; XX/XO GSD) *

Panagrellus (Free-living; gonochorists) *

Panagrolaimus (Free-living; parthenogenetic females) *

Steinernema (Entomopathogenic)

Rhabditophanes (Free-living; parthenogenetic females) *

S. papillosus (Obligate parasite with single free-living generation; parthenogenetic females \{parasitic\} \& gonochorists \{free-living\}; environmentally controlled CSD with chromatin diminution) *

S. ratti (Obligate parasite with single free-living generation; parthenogenetic females \{parasitic\} \& gonochorists \{free-living\}; environmentally controlled CSD with XX/XO) *

S. stercoralis (Obligate parasite with single free-living generation)

Parastrongyloides (Facultative parasite with consecutive free-living generations; gonochorist \{parasitic \& free-living\}; XX/XO GSD) *

Aphelenchoides (Plant parasite)

Bursaphelenchus (Plant parasite)

Meloidogyne (Plant parasite)

B

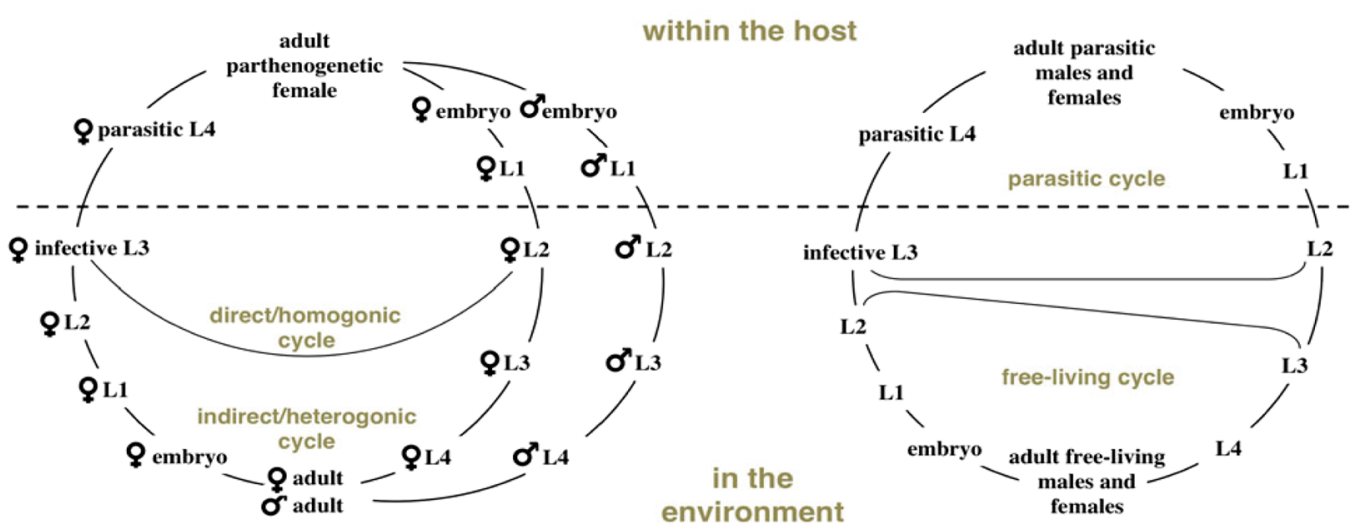

Strongyloides ratti

Parastrongyloides trichosuri

C Life cycle comparison between C. elegans and Strongyloides spp.

\begin{tabular}{|c|c|c|}
\hline Molt & C. elegans $\left(22^{\circ} \mathrm{C}\right)$ & $\begin{array}{c}\text { Strongyloides spp. } \\
\left(23-26^{\circ} \mathrm{C}\right)\end{array}$ \\
\hline L1 - L2* & $\sim 12 \mathrm{~h}$ & $4-10 \mathrm{~h}$ \\
\hline${\text { L2 }-\mathrm{L3}^{\star}}^{*}$ & $\sim 20 \mathrm{~h}$ & $12-14 \mathrm{~h}$ \\
\hline L3 - L4* & $\sim 28 \mathrm{~h}$ & $18-20 \mathrm{~h}$ \\
\hline L4 - Adult & $\sim 38 \mathrm{~h}$ & $\sim 26 \mathrm{~h}$ \\
\hline $\begin{array}{c}\text { Adult capable of laying } \\
\text { eggs }\end{array}$ & $\sim 46 \mathrm{~h}$ & $\sim 28 \mathrm{~h}$ \\
\hline
\end{tabular}

* Time estimates are post hatching in all cases 
Fig. 1 Introduction to Stronygloides nematodes. a A schematic cladogram to illustrate the phylogenetic position and interrelationships of Strongyloides with other nematode species based on Holterman et al. (2006). Species discussed in the text are marked with red asterisks. In brackets are the lifestyles and for the relevant species, their modes of reproduction (GSD genetic sex determination, CSD chromosomal sex determination, $X X / X 0$ sex determining system where females have two $\mathrm{X}$-chromosomes and males only one). Branch lengths on this cladogram are irrelevant. b The generalized life cycle of Strongyloides species (left) compared to the life cycle of Parastrongyloides trichosuri (right). In the text infective L3 larvae are abbreviated as L3i. c A comparison of the developmental timing between C. elegans (according to WormAtlas) and Strongyloides spp. from hatching (for C. elegans) or start of culture (deposition of feces by the host for Strongyloides spp.) to adulthood. For Strongyloides spp., molt times are in a wide range because not all embryos/larvae are of exactly the same age in freshly deposited feces and additionally due to the fact that males develop and molt faster in comparison to females

recently developed resources for working with Strongyloides spp. and their close relative Parastrongyloides trichosuri (P. trichosuri), a facultative parasite of Australian possums (Eberhardt et al. 2007; Grant et al. 2006a, b; Nemetschke et al. 2010b; Shao et al. 2012; Viney et al. 2002), render this group of parasites more experimentally utilizable. The nematode genera of Strongyloides and Parastrongyloides together constitute the family of Strongyloididae (Dorris et al. 2002).

\section{Life histories of $S$. ratti, $S$. papillosus, and P. trichosuri}

The life cycle of Strongyloides spp. (Fig. 1b) has been reviewed recently (Streit 2008; Viney and Lok 2015). The parasitic worms are all female and live in the small intestines of their respective hosts. They reproduce by mitotic parthenogenesis but nevertheless give rise to female and male offspring. These young female offspring have two lifestyle choices: they either develop into filariform third-stage infective larvae (L3i) and upon infection of a new host develop into parasitic adults (termed direct or homogonic development), or they develop by a rhabditiform L3 stage, along with all the males, to finally give rise to a facultative sexually reproducing free-living generation (termed indirect or heterogonic development). Offspring of the free-living adults are all female and bound to develop into parasites, with very few known exceptions (Streit 2008; Yamada et al. 1991). P. trichosuri is the best-studied representative of Parastrongyloides, a genus closely related to Strongyloides (Dorris et al. 2002). These species also form parasitic and free-living generations of reproducing adults. Nevertheless, their life history (Fig. 1b) and reproductive modes differ from those of Strongyloides spp. in interesting ways (Grant et al. 2006b). Firstly, parasitic males exist in Parastrongyloides (Mackerras 1959) and reproduction in this generation is sexual (Grant et al. 2006b; Kulkarni et al. 2013). Secondly, free-living Parastrongyloides spp. produce progeny of both sexes, and lastly, members of this genus ( $P$. trichosuri in particular) have been shown to undergo an unlimited number of consecutive free-living generations (Grant et al. 2006b), making it a facultative parasite. However, the life cycle of the free-living generation in both Strongyloides and Parastrongyloides is rather short, even in comparison to C. elegans (Fig. 1c).

\section{Sex determination}

In all species of Strongyloides investigated thus far, the sex ratio of the progeny produced by parasitic females is under the influence of the host's immune system (Streit 2008), with an increasing immune response against the worms leading to a higher proportion of males. Male and female Strongyloides worms normally differ in their chromosomes (Streit 2008). However, the finer details of the sex chromosomes may differ among species. For example, $S$. ratti females have two Xchromosomes, but males have only one, in addition to the two pairs of autosomes in both sexes. Hence, $S$. ratti employs an environmentally influenced $\mathrm{XX} / \mathrm{X} 0$ sex determination with $2 n=6$ in females and $2 n=5$ in males (Harvey and Viney 2001; Nigon and Roman 1952). In S. papillosus, the genetic material homologous to $S$. ratti chromosomes $\mathrm{X}$ and I is combined into one large chromosome (Nemetschke et al. 2010a). In the males of this species, sex-specific chromatin diminution creates a hemizygous region largely corresponding in sequence to the X-chromosome in S. ratti. Presumably, this chromatin diminution event helps to functionally restore the ancestral XX/X0 sex determining system (Albertson et al. 1979; Kulkarni et al. 2013; Nemetschke et al. 2010a). During this process, an internal portion of one chromosome is eliminated but both ends are retained as separate chromosomes, leading to the $2 \mathrm{n}=5$ in male and $2 \mathrm{n}=4$ in female karyotypes. By contrast, P. trichosuri employs chromosomal $\mathrm{XX} / \mathrm{X} 0$ sex determination with $2 n=6$ in females and $2 n=5$ in males. In this species, there is no indication for an environmental influence on sex determination (Grant et al. 2006b; Kulkarni et al. 2013).

\section{The germline in the nematode family of Strongyloididae}

The gonads in both sexes of the model nematode C. elegans are essentially tubular (Hubbard and Greenstein 2005). The hermaphroditic gonad has two arms, one extending anteriorly and one posteriorly, with both arms terminating in a central vulva. In the males, the gonad has just one arm with a posterior opening. A somatic cell, termed the distal tip cell (DTC) sits at the very tip of each gonad arm and signals the nearby germ cells to proliferate mitotically via Delta/Notch signaling (Kimble 
and Crittenden 2005). Once cells move out of the reach of the DTC signal, they exit the mitotic cell cycle and initiate meiosis, thereby beginning their differentiation into gametes. Hence, C. elegans, like many other nematodes (Rudel et al. 2005; Rudel and Sommer 2003), maintains a stem cell population at the distal end of each gonad arm and this creates a constant flow of increasingly differentiated germ cells from the distal tip to the proximal end (Fig. 2 (top), C. elegans germline and insets 1-4). In addition, all cells in the distal portion of the gonad, which is situated at the dorsal side of the adult worm, open into a common central rachis and form a large syncytium.

While the overall morphology of the gonad is very similar to that of C. elegans, the appearance and organization of the germ cells differ greatly in $S$. ratti and other members of the Strongyloididae (Fig. 2, S. ratti germlines and insets 1-4) to that of C. elegans (Hammond and Robinson 1994; Triantaphyllou and Moncol 1977). For S. ratti, the distal arm was shown to contain giant nuclei, which have been reported to have a DNA content of up to several hundred C (Fig. 2, $S$. ratti inset 1), with $1 \mathrm{C}$ being the DNA content of a haploid set of chromosomes (Hammond and Robinson 1994). The distal arm (i.e., the region with the giant nuclei) is followed by a band of very small, compact nuclei at the gonadal loop (Fig. 2, S. ratti inset 2), proximal to which presumably meiotic nuclei with condensed chromosomes can be observed (Fig. 2, S. ratti insets 3 and 4). Further along the gonad, differentiated oocytes or sperm (depending on the sex) are present, very similar to C. elegans.

Other than these basic morphological aspects, information regarding germline development and gene expression for Strongyloididae members is currently lacking. For C. elegans, many aspects of the control of germline development and gene expression have been shown to act through chromatin modifications, with these being extensively studied and well characterized (Kimble and Crittenden 2005; Schaner and Kelly 2006). Histone modifications have also been shown to be important determinants for establishing transcriptionally active or inactive domains in different organisms (Rando 2012), and therefore are an integral part of germline regulation.

Here, we present the first detailed comparative characterization of the germlines of free-living males and females of Strongyloididae (S. ratti, S. papillosus, and P. trichosuri) and compare this with $C$. elegans. We confirm that in the adult gonad, the distal arm is occupied by giant nondividing polyploid nuclei, and that there are no mitotically proliferating stem cells here, despite the presence of a DTC-like cell in these species. Additionally, it appears that proliferation of the germ nuclei is restricted to early and mid-larval development. Further, we describe differences in the germline chromatin of Strongyloididae members (especially in $S$. ratti) and C. elegans, by using conserved histone modifications with a particular emphasis on Histone3 phosphorylated at serine 10 (H3Pser 10) and Histone 3 tri-methylated at lysine 4 (H3K4me3). The variations in these histone modifications are further explored through comparisons with three other nematode species (Rhabditophanes KR3021, Panagrolaimus PS1159, and Panagrellus PS1163), each of which are at an increasing phylogenetic distance to $S$. ratti, unveiling further complexities in germline development among nematodes. Finally, we discuss the possible implications this has on regulating cell cycles and active transcription for Strongyloididae members.

\section{Materials and methods}

\section{Culturing and manipulating nematodes}

S. ratti ED321 and S. papillosus isolate LIN were maintained as described (Eberhardt et al. 2007; Nemetschke et al. 2010b; Viney et al. 1992). All animal experimentation was done according to national and international guidelines, with local authorities granting the required permits. P. trichosuri was cultured in continuous free-living cycles (Grant et al. 2006b) at $20^{\circ} \mathrm{C}$ on NGM plates seeded with Escherichia coli (E. coli) OP 50 bacteria (Stiernagle 1999) supplemented with a piece of autoclaved rabbit feces. C. elegans N2, Panagroliamus PS 1159, Panagrellus PS1163, and Rhabditophanes KR3021 were all maintained on NGM plates seeded with $E$. coli OP 50 bacteria at $20^{\circ} \mathrm{C}$, with the exception of Rhabditophanes KR3021 which was kept at $15^{\circ} \mathrm{C}$.

\section{DAPI staining/microscopy}

Adult worms (of the desired age) were fixed with ice-cold $100 \%$ methanol (Roth GmbH and Co. KG) and directly mounted (without a rehydration series) on polylysine-coated glass slides in $10 \mu \mathrm{L}$ of Vectashield (Vector Laboratories Inc., Burlingame, CA 94010) containing $1 \mu \mathrm{g} \mathrm{mL}^{-1}$ DAPI (4',6diamidino-2-phenylindole from Roche). Confocal stacks of the entire gonad were taken for manual counting of nuclei and processed in ImageJ (Fiji). For dissected gonad DAPI imaging, the same protocol was followed after gonad dissection.

\section{Gonad staging}

Worms were collected by the Baermann funnel method every $2 \mathrm{~h}$ after hatching as described by Basir (1950). Each worm sample was split into two, one was directly viewed under the differential interference contrast (DIC) microscope and the other was used for DAPI staining and confocal imaging as described above. 


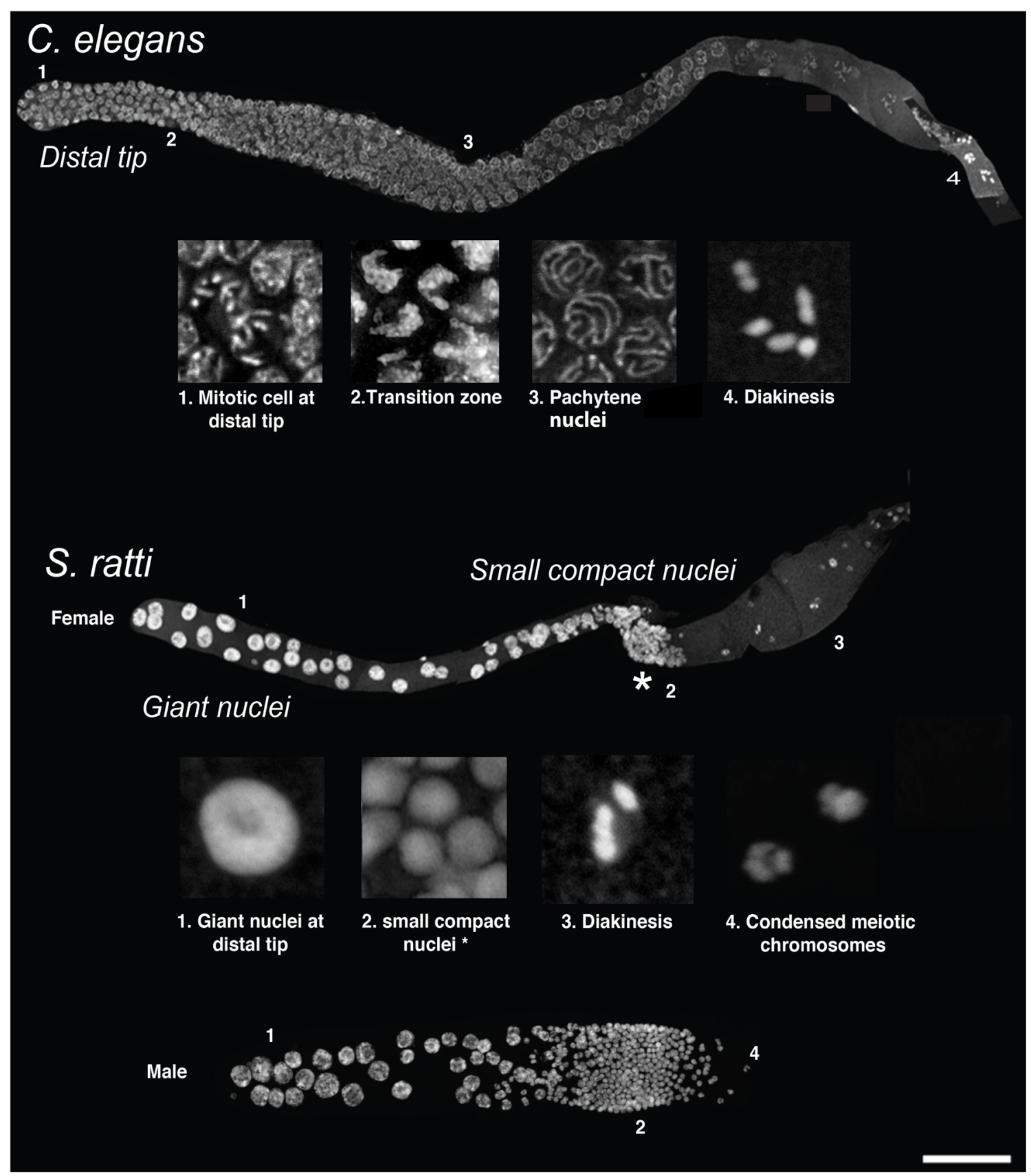

Fig. 2 Comparisons between the C. elegans and Strongyloides gonads. Top: a DAPI-stained dissected C. elegans hermaphroditic (adult) gonad, showing progression of germ cells in the germline (distal tip is to the left). The numbers 1-4 indicate the immediate insets below, with each inset showing the characteristic morphology (mitotically dividing cells at distal tip, crescent-shaped nuclei at transition zone, "bowl of spaghetti" in the pachytene zone and condensed chromosomes at diakinesis, respectively) of germ nuclei for those regions. Bottom: DAPI-stained dissected gonads from $S$. ratti adult females (top) and males (bottom) showing the completely different gonadal organization in comparison to C. elegans. Note the shorter but broad nature of the $S$. ratti male gonad in comparison

\section{Transmission electron microscopy of $S$. papillosus gonads}

Samples were cryo-fixed with a Baltec HPM-010 high-pressure freezer and were freeze-substituted in a Leica AFS-2 to the female (adult males are smaller in size to adult females; adults are approximately $28-30 \mathrm{~h}$ post-culturing). Here, the entire distal arm is occupied by intensely staining giant nuclei, followed by a band of small compact nuclei at the gonadal loop (asterisk). Except for even more strongly condensed small nuclei in males, the organization is identical in both sexes. Insets 1 and 2 are derived from female gonads. The band of small nuclei is followed proximally in females by nuclei, which might be in diakinesis (shown in inset 3 taken from a female germline) and in males with condensed presumably meiotic chromosomes (inset 4, taken from a male germline). Scale bar $50 \mu \mathrm{m}$

according to following protocol: $56 \mathrm{~h}$ at $-90{ }^{\circ} \mathrm{C}$ in acetone with $5 \%$ gallic acid monohydrate (Roth, Karlsruhe, Germany), $3 \mathrm{~h}$ warmed up to $-60{ }^{\circ} \mathrm{C}, 5 \times$ washed with prechilled acetone, $24 \mathrm{~h}$ at $-60{ }^{\circ} \mathrm{C}$ with $2 \% \mathrm{OsO}_{4}, 0.5 \% \mathrm{UA}, 0.5$ 
GA, $2 \% \mathrm{H}_{2} 0$, warmed up to $0{ }^{\circ} \mathrm{C}$ at $4{ }^{\circ} \mathrm{Ch}^{-1}, 5 \times$ washed with pre-chilled acetone and infiltrated over a total period of $29 \mathrm{~h}$ with increasing concentrations of epoxy resin (EMbed-812kit, Science Services, Munich, Germany); finally, the samples were cured at $60{ }^{\circ} \mathrm{C}$ for $48 \mathrm{~h}$ in flat embedding molds. Longitudinal semithin sections were stained with osmium tetraoxide and viewed in a FEI Tecnai $\mathrm{G}^{2}$ Spirit transmission electron microscope operating at $120 \mathrm{kV}$. Images were taken with a Gatan Ultrascan 4000 camera at maximum resolution using the manufacturer's software.

\section{BrdU labeling of $C$. elegans, $S$. ratti, and P. trichosuri germlines}

5-Bromo-2'-deoxyuridine (BrdU) labeling of germlines was done as described by Crittenden et al. (2006), and dissected germlines were visualized at different time points $(3,6,12$, and $16 \mathrm{~h}$ ) by staining against an anti-BrdU-FITC-labeled antibody (BD Biosciences). For Strongyloides spp., males and females of various stages were manually selected and put on plates seeded with labeled $E$. coli and incubated for 3, 6, 12, or $16 \mathrm{~h}$ (in order to start the labeling with very young larvae, gravid females were allowed to lay their eggs on labeled E. coli plates). At the end of this time period, the larval stage the individuals had reached was scored and the germlines were dissected and used for antibody staining. Young Strongyloides larvae with prolonged exposure to labeled E. coli plates $(<16-18$-h exposure) were found to be visibly very sick or dead.

\section{Immunostaining of nematode species}

Gonads from adult worms were dissected in egg buffer (118 mM NaCl, $48 \mathrm{mM} \mathrm{KCl} 2,2 \mathrm{mM} \mathrm{CaCl} 2,2 \mathrm{mM} \mathrm{MgCl} 2$, $5 \mathrm{mM}$ HEPES) containing $0.1 \%$ Tween and immediately fixed in $1 \%$ paraformaldehyde for $5 \mathrm{~min}$. Slides were frozen in liquid nitrogen, freeze-cracked, and then immersed for $1 \mathrm{~min}$ in methanol at $-20{ }^{\circ} \mathrm{C}$ and transferred to PBS-Triton $\mathrm{X}-100$ (1× PBS, $1.5 \%$ Triton X-100). Blocking in $0.7 \%$ BSA in PBS-Triton X-100 was then carried out for $1 \mathrm{~h}$. Primary antibodies were incubated overnight at room temperature, slides were then washed three times for $10 \mathrm{~min}$ in PBSTriton X-100, and secondary antibodies were added and incubated for 4-6 h at room temperature. Following three washes for 5 min in PBS-Triton X-100, the slides were counterstained in $10 \mu \mathrm{L}$ of Vectashield (Vector Laboratories Inc.) containing $1 \mu \mathrm{g} \mathrm{mL} L^{-1}$ DAPI (Roche). The following primary antibodies were used at the indicated dilutions: Anti-BrdU-FITC labeled (1:2.5, BD Biosciences), rabbit H3K9/K14ac (1:500, Diagenode), rabbit H4K20me1 (1:500, Diagenode), mouse H3K27ac (1:500, Diagenode), mouse H3K27me3 (1:500, Diagenode), rabbit H3K9me1 (1:500, Diagenode), rabbit H3Pser10 (1:200, Millipore), mouse H3K4me3 (1:500,
Diagenode), and rabbit $\alpha$-tubulin (1:200), rabbit $\alpha$-Actin $(1: 200)$. The following secondary antibodies were used for visualization: Goat anti-rabbit labeled with Alexa Fluor 488 (1:200), goat anti-mouse labeled with Cy3 (1:200), and mouse anti-biotin labeled with Cy3 (1:200).

All images were acquired as a stack of optical sections with an interval of $0.65 \mu \mathrm{m}$ using an Olympus confocal FV1000 microscope and processed in ImageJ (Fiji) and Adobe Photoshop CS.5.

\section{FISH in $S$, ratti}

Gonads from adult worms were dissected in egg buffer (118 mM NaCl, $48 \mathrm{mM} \mathrm{KCl2,} 2 \mathrm{mM} \mathrm{CaCl} 2,2 \mathrm{mM}$ $\mathrm{MgCl} 2,5 \mathrm{mM}$ HEPES) containing $0.1 \%$ Tween and immediately fixed in $1 \%$ paraformaldehyde for $5 \mathrm{~min}$. Slides were frozen in liquid nitrogen, freeze-cracked, and then immersed for $1 \mathrm{~min}$ in methanol at $-20{ }^{\circ} \mathrm{C}$ and then transferred to $2 \times \mathrm{SSCT}$ three times for $5 \mathrm{~min}$ each. Then, the slides were dehydrated for 3 min each in 70, 90, and $100 \%$ ethanol after which the slides were left to airdry. After air-drying, the slides were ready for adding the hybridization mix containing the florescent in situ hybridization (FISH) probes. The amount of probe per slide was between 150 and $200 \mathrm{ng}$. The final concentration of the hybridization mix after adding the probe was $2 \times$ SSCT, $50 \%$ formamide, $10 \% \mathrm{w} / v$ dextran sulfate. Fifteen microliters of this mix was added per slide followed by heating the slides at $93{ }^{\circ} \mathrm{C}$ for $2 \mathrm{~min}$ in a heated block. The slides were then removed and incubated overnight at $37^{\circ} \mathrm{C}$ in a humid chamber. For post-hybridization washes, the slides were rinsed in $2 \times$ SSCT $50 \%$ formamide at $37{ }^{\circ} \mathrm{C}$ for $30 \mathrm{~min}$. This was followed by three washes in $2 \times$ SSCT for 5 min each. Then, the slides were blocked in $1 \% \mathrm{BSA}$ in $2 \times \mathrm{SSCT}$ for $30 \mathrm{~min}$. Then, $50 \mu \mathrm{L}$ of the primary H3Pser 10 antibody was added per slide and incubated overnight at room temperature in a humid chamber. Then, slides were washed in $2 \times$ SSCT, three times for $10 \mathrm{~min}$ each, and incubated with $50 \mu \mathrm{L}$ of the labeled secondary antibody (1:200) for 4-6 h. Finally, the slides were washed three times in $2 \times$ SSCT for 10 min each and were counterstained in $10 \mu \mathrm{L}$ of Vectashield (Vector Lab. Inc.) containing $1 \mu \mathrm{g} \mathrm{mL}^{-1}$ DAPI (Roche) and ready for imaging using the Olympus confocal FV1000 microscope.

\section{Preparation of FISH probes}

Probes (22-25 bp) were ordered from Eurofins MWG Operon (after ensuring preferably a single hit in the genome on the required chromosome). The ready-made probes were labeled internally with biotin along their entire length. They were visualized using a mouse monoclonal anti-biotin $\mathrm{Cy} 3$ antibody from Sigma-Aldrich. 


\begin{tabular}{llll}
\hline S. ratti FISH probe & Sequence & Chromosome and position & $y t P$ marker/contig information \\
FISH-1 & CGATCCATTCAAAAAGAAAGCTGAA & Autosome II at $-4.1 \mathrm{cM}$ & ytP91 \\
FISH-2 & CTGAACTTCAAGCAGAATTACGTGAAG & Autosome II at $-4.1 \mathrm{cM}$ & $\mathrm{ytP91}$ \\
FISH-3 & CTTACTTTGGATAAATCATTTAA & Autosome II at $0.0 \mathrm{cM}$ & $\mathrm{ytP113}$ \\
FISH-4 & GAATATTGACCGTTGCTGGATCTTTA & Autosome I at $0.0 \mathrm{cM}$ & $\mathrm{ytP37}$ \\
FISH-5 & TTGCCGGAGTTCCGACAATGGGAG & Autosome I at $0.0 \mathrm{cM}$ & ytP37 \\
FISH-6 & TGCTATGAAAGCTGGTTGGAAACAA & Autosome I at $2.2 \mathrm{cM}$ & ytP117 \\
FISH-7 & ATTAAAATTACTGATAAATAACTC & Chromosome X & S.ratti_chrx_000001 \\
FISH-8 & AGCAGAATATAAAAGGAAGAACAAACTG & Chromosome X & S.ratti_chrx_000001 \\
FISH-9 & GTTATTTTCTATTAAAGACGGTGAAGA & Chromosome X & S.ratti_chrx_000001 \\
\hline
\end{tabular}

\section{Results}

\section{Timing of germ cell proliferation in Strongyloididae}

Simple cytological observation has revealed no obvious mitotic cell divisions in the germline of the adult free-living Strongyloides spp., implying that these organisms do not maintain a population of proliferating stem cells in their adult germlines. In order to test this hypothesis, we first performed a detailed electron and light microscopic characterization of the distal gonad of $S$. papillosus adult females (Fig. 3) and males (data not shown). Given the high degree of similarity in the organization of the gonads between $S$. papillosus, $S$. ratti, and $P$. trichosuri, we assume that this morphological description holds true for the latter species as well. Detailed cytological examination using the transmission electron microscope (TEM) found no indication for mitotic activity in any part of the germline, for either $S$. papillosus males or females. Nevertheless, a DTC was observed, which assumed a somewhat different position than in C. elegans or Pristionchus pacificus (Fig. 3b, c). Most notably, it appears that this DTC-like cell, which is noticeably smaller, may sit lop-sided in these species and does not necessarily cap the whole distal tip as it does in C. elegans or P. pacificus (Fig. 3d). We also observed processes of the DTC-like cell making contact internally with the gonad at regular intervals along the entire distal arm (Fig. 3e, f). Furthermore, we found the cytoplasm of the distal arm to be very rich in ribosomes and mitochondria (Fig. 3g).

In order to then determine when germ cell proliferation occurs and to ascertain the development of the germline at different larval stages, we stained different developmental stages of $S$. papillosus, S. ratti, and P. trichosuri with DAPI and additionally performed bromo-deoxyuridine (BrdU) incorporation experiments (BrdU uptake indicates actively cycling cells in $\mathrm{S}$ phase) for these species with $C$. elegans as a control. We first came up with a schematic for how the germline grows in these species, by correlating the development of the gonad at different larval molts under the differential interference contrast (DIC) microscope and with DAPI staining (Fig. 4a). In both species of Strongyloides and in P. trichosuri, we observed mitotic figures only in the germlines of larvae L3 and younger under DAPI staining (Suppl Fig. 1A). While such an in-depth analysis has not been carried out before, our results nevertheless corroborate earlier reports (Triantaphyllou and Moncol 1977), confirming no cellular divisions in the adult germlines of either sex. In addition, we observed that the giant nuclei first appeared in L3 larvae, coinciding with the arrest of all detectable mitotic germline activity in the distal arm. This is consistent with our observation of no BrdU in the adult giant or small nuclei, for animals that had been exposed to BrdU only after reaching the fourth larval stage (Fig. 4b). However, BrdU was incorporated into all the germline nuclei for animals that were exposed to it as younger larvae (SupplFig. 1B). BrdU was also readily detected in the germ nuclei of $C$. elegans control animals that were treated as L4 larvae and adults.

\section{Position of the central rachis and germline fluid dynamics in $S$. papillosus and $P$. trichosuri}

As the central rachis and syncytium is so prominent in the structure of the $C$. elegans germline (Hubbard and Greenstein 2005), we were interested in looking at the presence, position, and possible function of the central rachis in Strongyloides species. Using a combination of TEM and staining against $\alpha$-tubulin (Fig. 5a), we found that the central rachis in these species begins just prior to the band of small compact nuclei before the gonadal loop region (in females), instead of running all along the distal arm as in C. elegans. In males, the rachis is slightly less pronounced, with it being detected at the base of the band of small compact nuclei (data not shown).

Due to the gonadal structure and function in C. elegans, there is a constant flow of cytoplasm from the distal tip toward the proximal end of the gonad, indicating active transport. If small lipid droplets are injected at any place in the distal gonad, they are transported 



Fig. 3 Transmission electron microscopy (TEM) of the S. papillosus free-living female gonad. a Semithin longitudinal TEM sections of a $S$. papillosus female showing the entire gonad (outlined in red) in the body of the adult worm, with the vulva (top, central). The two distal gonad arms are marked as DG1 and DG2 (distal tips are marked by asterisks); the gonadal loop is labeled as GL and the proximal gonad as PG. Female adults that were approximately $28-30 \mathrm{~h}$ post-fecal culturing were used for this analysis. b TEM section showing the distal tip of the gonad, $D G$, with giant nuclei, $G N$ (outlined in blue), and the distal tip cell, DTC (in yellow). c Zoom in of the DTC (yellow) showing its nucleus, $D T C N$ (in red) in addition to the giant nuclei, GN (in blue) in the distal gonad, DG. d TEM section from Pristionchus pacificus showing the DTC (outlined in yellow) sitting as a cap on the distal tip (outlined in gray), with germ cells GC, around a central rachis "rc" (in red). This organization is similar to what is found in C. elegans (image courtesy of Metta Riebesell). e The distal tip cell and its processes (outlined in yellow) making contact with the distal gonad at regular intervals. $\mathbf{f}$ Magnified view showing a DTC process (in yellow) contacting the distal gonad close to two giant nuclei GN (in blue). $\mathbf{g}$ Zoom in at the distal tip (with DTC outlined in yellow) showing the high density of ribosomes (electron dense regions) and mitochondria, $M$ (outlined in white) in the distal gonad, $D G$ proximally via the central rachis in the gonad, where-upon they are incorporated into growing oocytes (Wolke et al. 2007). As the central rachis in Strongyloides species was distinctively different in position, we performed the same experiment in S. papillosus and in P. trichosuri (Fig. 5b), obtaining in part similar results to $C$. elegans. While droplets placed just before the gonadal loop or at the loop moved proximally and were rapidly incorporated into 

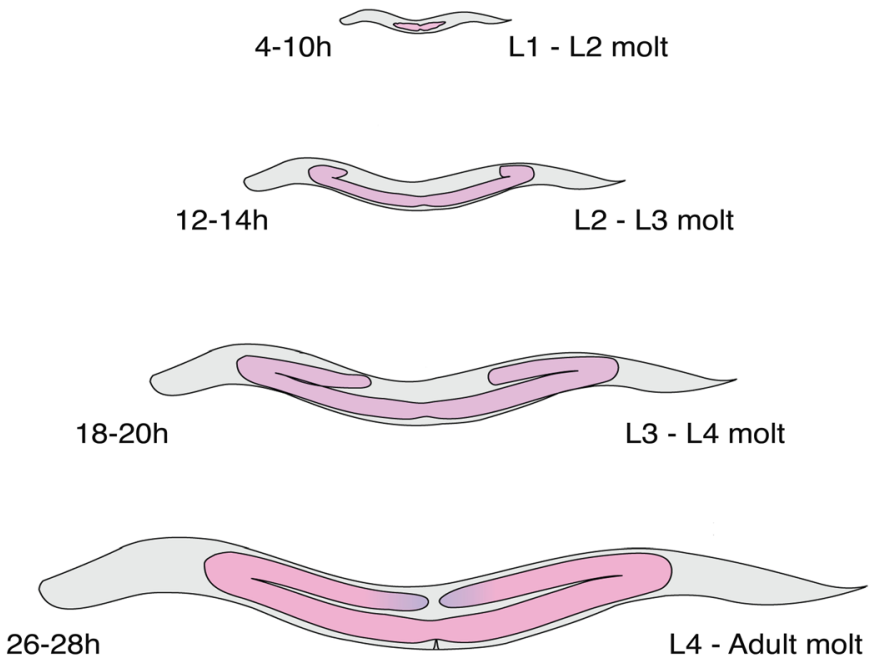

B

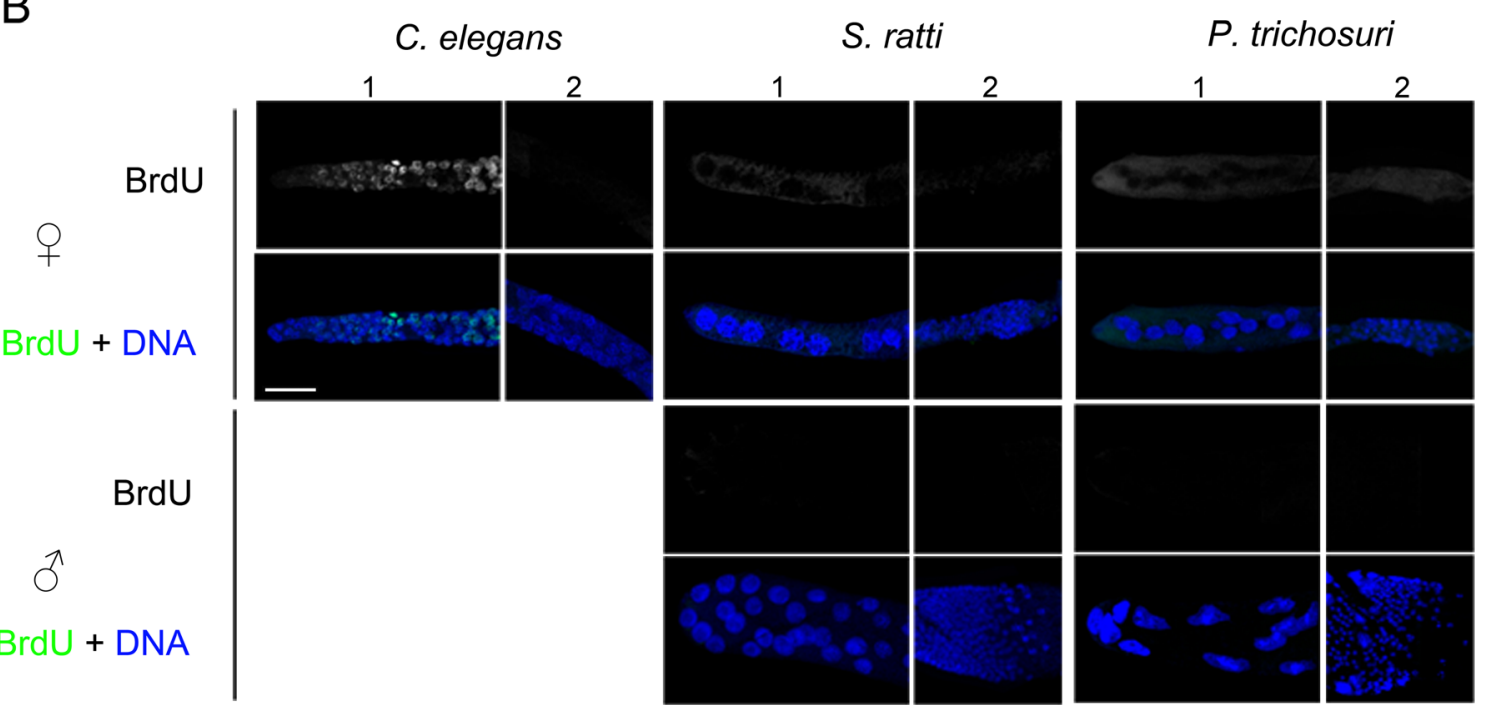

Fig. 4 a Gonad size and shape at the time of the different molts. A cartoon representation of the worm is outlined in gray and the germline is represented in pink (with the distal tip in purple in L4 adults). The time post-feces deposition/culturing is indicated in hours to the left, with suitable approximations made for molt timings for the two Strongyloides species. The different larval molts are indicated to the right. For all stages anterior is to the right and ventral is below. b Bromo-deoxyuridine (BrdU) incorporation assays in C. elegans, $S$. ratti, and $P$. trichosuri adult germlines. Panel 1 represents the distal gonad (with giant nuclei in $S$. ratti and P. trichosuri) and panel 2 the gonadal loop region (with small nuclei in $S$. ratti and $P$. trichosuri). The top two rows show female (hermaphrodites in the case of C. elegans), and

growing oocytes, droplets injected along the distal arm were not transported at all in these species. This supports our microscopic observations (Fig. 5a) and confirms that a functional central rachis starts just prior to the gonad loop in these species. the two bottom rows show male germlines. The images were taken $3 \mathrm{~h}$ post-BrdU exposure. For both females and males, the BrdU channel is shown as separate in gray (top) or as merged with DNA (BrdU in green, DNA in blue). In C. elegans hermaphrodites, BrdU is seen incorporated into mitotically active cells in the proliferative zone at the distal tip of the germline $3 \mathrm{~h}$ post-BrdU exposure (C. elegans, panel 1). In comparison, no detectable BrdU uptake is seen within giant nuclei or the small compact nuclei in S. ratti and P. trichosuri (in either sex) indicating no active DNA replication in these regions in adults. Similar results were obtained at 6,12 , and $16 \mathrm{~h}$ post-exposure to BrdU in these two species, although a cytoplasmic signal was often seen. Scale bar $20 \mu \mathrm{m}$

\section{Meiotic progression and histone modifications in the germlines of $S$. ratti}

Until now, nuclei in Strongyloides spp. were only recognized as meiotic once they had cytologically observable 
A

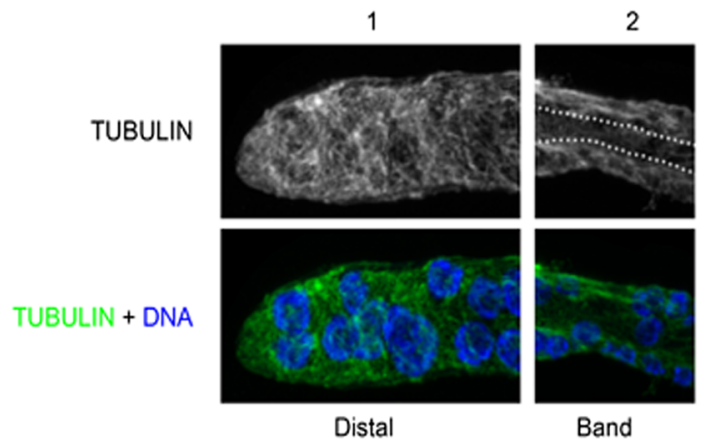

B

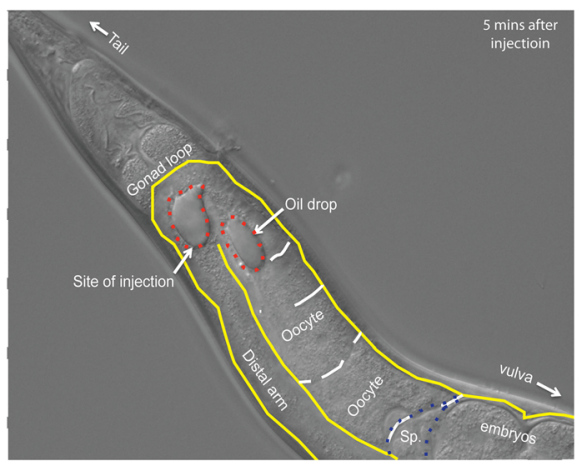

Fig. 5 Central core and fluid dynamics. a The central core in $S$. papillosus dissected female gonads visualized by staining against $\alpha$ tubulin (green) and DAPI (blue). Panel 1 shows no visible central canal in the distal gonad (with the giant nuclei) when stained with $\alpha$-tubulin (top), but a clearly visible canal in panel 2 (outlined in white) starting just before the gonadal loop (beginning of the band of small nuclei). Bottom panels show merge with DAPI. b Differential interference contrast (DIC) timelapse images of an injection experiment using mineral oil in a $P$. trichosuri adult female gonad, showing the rapid movement and incorporation of an oil droplet (if injected into the central core) proximally into growing oocytes. The gonad arm is outlined in yellow, the oil drop in red, developing oocytes are labeled and outlined in white dashes, the spermatheca (Sp.) is in blue, embryos labeled in the uterus outlined with green. Soon after injection, the single large oil drop is broken into smaller droplets, which quickly move proximally past the gonadal loop (seen in the left most image marked as the oil drop, at 5 min postinjection). Once proximal, the oil droplets move further down into developing oocytes (seen in the middle image at 20 min post-injection) to finally become incorporated within them (seen in the right most image, $1 \mathrm{~h}$ post-injection) condensed bivalent chromosomes (Nigon and Roman 1952; Triantaphyllou and Moncol 1977). In an attempt to determine the actual position of the onset of meiosis in the $S$. ratti germline, we used antibodies that had in other systems (particularly in C. elegans) been shown to be meiosis specific. We stained dissected gonads of $S$. papillosus and S. ratti (both sexes) with anti-RAD-51 (Rinaldo et al. 2002) and anti-REC-8 (Pasierbek et al. 2003) antibodies, along with anti-SMC-3, which localizes to both meiotic and mitotic cells (Chan et al. 2003). In order to test if these commercially available antibodies might recognize the corresponding Strongyloides proteins, we also performed Western blot analyses. Although all three antibodies did recognize single defined bands of the expected size in Western blots (Suppl Fig. 2A), no meaningful immunostaining patterns were obtained with any of these antibodies in the Strongyloides germline (Suppl Fig. 2B, data shown for only S. ratti RAD-51 stainings). For the moment, it must remain open if RAD-51, REC-8, and SMC-3 are indeed not present in Strongyloides gonads or if the heterologous antibodies we used are not suitable to detect these proteins in situ for these species. In order to overcome this problem, we focused on antibodies against conserved histone modifications (Suppl Fig. 3A). We chose two histone modifications for comparison, one of which in C. elegans marks mitotically dividing germ nuclei ( $\mathrm{H} 3$ phosphorylated at serine 10, H3PSer10) and the other marking transcriptionally active chromatin regions ( $\mathrm{H} 3$ tri-methylated at lysine 4, H3K4me3), respectively (Hsu et al. 2000). Interestingly, while we did detect the published pattern in our C. elegans control (Hsu et al. 2000), H3Pser10 was completely absent from the distal gonad arm in $S$. ratti females (Fig. 6, S. ratti and C. elegans panels). Nevertheless, it was detected in the band of small compact nuclei, in a diffused and weak pattern (Fig. 7, S. ratti and C. elegans panels). In comparison, a very strong $\mathrm{H} 3 \mathrm{~K} 4 \mathrm{me} 3$ signal was seen in the giant nuclei along the entire distal arm and in the small compact nuclei in female $S$. ratti. Additionally, it appears that $\mathrm{H} 3 \mathrm{~K} 4 \mathrm{me} 3$ assumes a subnuclear localization that was mutually exclusive to the H3Pser10 signal in the small compact nuclei (Fig. 7, S. ratti panel and inset). By contrast, in $C$. elegans, $\mathrm{H} 3 \mathrm{~K} 4 \mathrm{me} 3$ was weak but 
Female distal gonad (giant nuclei) panel

H3K4me3

S. ratti

P. trichosuri
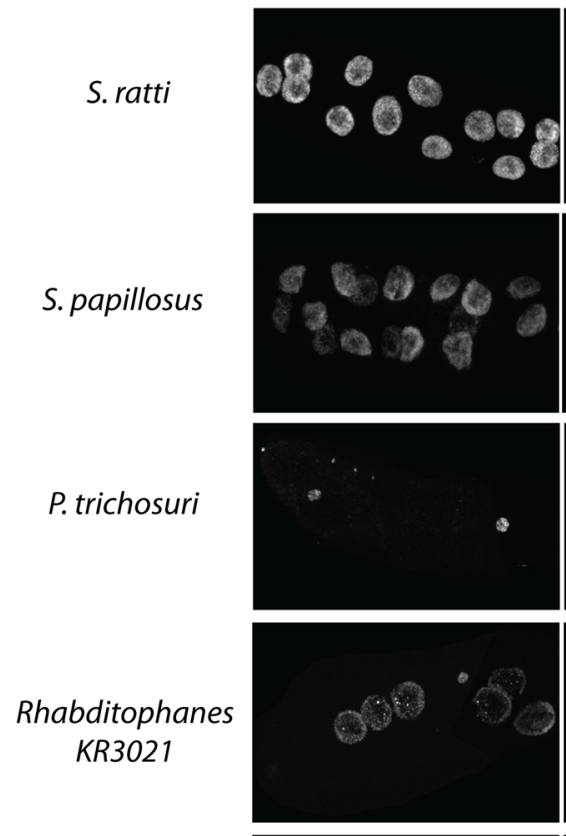

H3Pser10
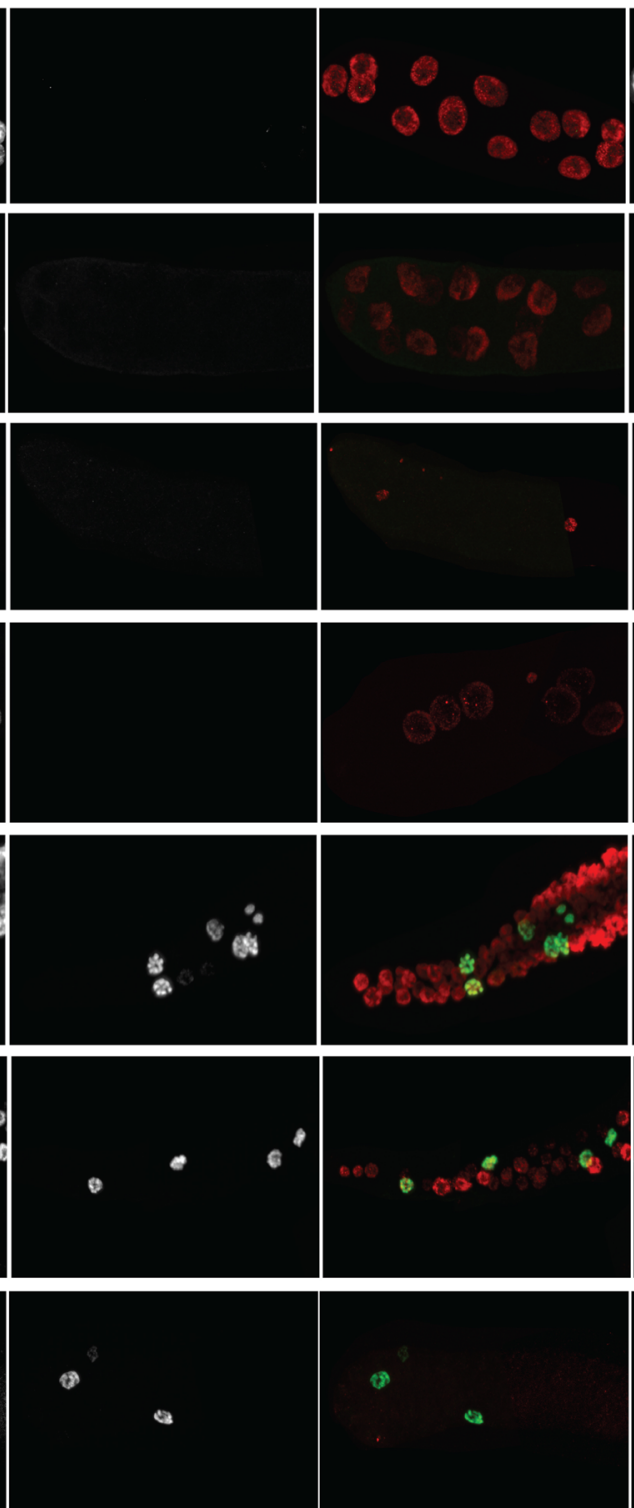

DAPI
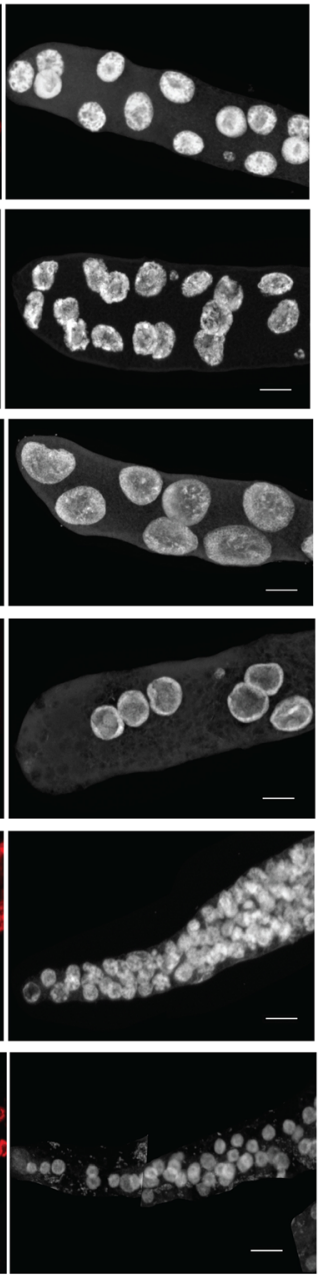

(⿻)

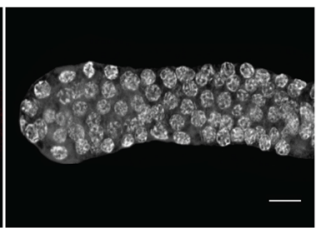

Fig. $6 \mathrm{H} 3 \mathrm{~K} 4 \mathrm{me} 3$ and H3Pser10 staining patterns in females of different nematode species. H3K4me3 (in red) and H3Pser10 (in green) antibody stainings (with the individual channels separated according to color and labeled on top) in seven different (adult female) nematode species in the distal part of their gonads (distal tip is to the left for each). This region consists of giant nuclei in Strongyloides species. Note the similarity in gonad organization in $S$. ratti, S. papillosus, P. trichosuri, and

detectable at the distal tip and fairly strong in pachytene nuclei in accordance to previous literature (Schaner and Kelly 2006).

\section{Evolutionary significance of histone modifications}

The dramatic differences in the germline histone modifications observed between $S$. ratti and C. elegans may be either
Rhabditophanes KR3021, and between Panagrolaimus PS1159, Panagrellus PS1163, and C. elegans. For nematodes with a gonad organization similar to $S$. ratti, note the lack of H3Pser10 staining (in green) in the distal gonad, whereas its presence in species with a gonad organization similar to C. elegans. Scale bar $10 \mu \mathrm{m}$ (note: adults are approximately 28-30 h post-culturing for Strongyloides species, but for other nematodes were young females carrying eggs in their uteri)

associated with the structural and functional differences in the germlines of these two species, or because of their large phylogenetic distance. In order to ascertain the evolutionary significance of these differences, we performed similar stainings using anti-H3Pser10 and anti-H3K4me3 on dissected gonads of five other clade IV nematode species ( $S$. papillosus, P. trichosuri, Rhabditophanes KR3021, Panagrolaimus PS1159, and Panagrellus PS1163). These species differ in 
Female gonadal loop (small nuclei) panel



Fig. $7 \mathrm{H} 3 \mathrm{~K} 4 \mathrm{me} 3$ and $\mathrm{H} 3$ Pser10 staining patterns in females of different nematode species. A comparative image showing H3K4me3 (in red) and H3Pser10 (in green) staining patterns (individual channels are separated according to color and labeled on top) obtained in the same seven nematode species in the gonadal loop region (for orientation, distal gonad arm is to the left for each). This region consists of the small compact nuclei in Strongyloides species. Insets (extreme right) are

their gonad organization such that $S$. papillosus, P. trichosuri, and Rhabditophanes KR3021 resemble the gonad organization in S. ratti, while Panagrolaimus PS1159 and

magnified views of the indicated regions that are marked in white in the merge panels, showing co-localization patterns of $\mathrm{H} 3 \mathrm{~K} 4 \mathrm{me} 3$ and H3Pser10 in each species. Note the localized dot-like H3Pser10 staining in the nuclei of $S$. ratti and Panagrellus PS1163, but its even distribution in the nuclei in S. papillosus and Panagrolaimus PS1159. H3Pser10 is absent in P. trichosuri (signal is from somatic sheath cells), Rhabditophanes KR3021 and C. elegans. Scale bar $10 \mu \mathrm{m}$

Panagrellus PS1163 are similar to C. elegans. Additionally, these species are of varying phylogenetic distance to $S$. ratti, while being equally distant from the clade $\mathrm{V}$ nematode 
C. elegans (Figs. 6 and 7; for nematode phylogenies, see Blaxter et al. (1998) and Holterman et al. (2006). We limited our analysis to females because some of the species involved were parthenogens. Although we observed striking differences between the species we studied, we noticed some general trends in staining patterns (Figs. 6 and 7 and Table 1). If species shared a gonad organization resembling that of $S$. ratti (i.e., S. papillosus, P. trichosuri, and Rhabditophanes KR3021), then they did not show any H3Pser10 staining in the distal gonad (Fig. 6, top four panels). Conversely, the freeliving clade IV members including Panagrolaimus PS1159 and Panagrellus PS1163, which have an overall germline organization more comparable with $C$. elegans, showed H3Pser10 staining in what appeared to be dividing germ cells in the distal gonad, just like in C. elegans (Fig. 6, bottom three panels). On the other hand, strong H3K4me3 staining extended up to the distal tip of the gonads of all clade IV members analyzed, with the notable exception of $P$. trichosuri, which did not show either H3K4me3 or H3Pser10 staining in the distal gonad (the few signals obtained for both antibodies are from nuclei of the somatic gonad; also note that all images shown are projections and not individual focal planes). Three (S. papillosus, Panagrolaimus, and Panagrellus) of the five clade IV species showed H3Pser10 staining in the gonadal loop region, just like in $S$. ratti (Fig. 7). Additionally, while in S. ratti and Panagrellus PS1163, the H3Pser10 signal was concentrated in a small region and the $\mathrm{H} 3 \mathrm{~K} 4 \mathrm{me} 3$ and H3Pser10 signals appeared mutually exclusive, in S. papillosus and Panagrolaimus PS1159, the areas occupied by H3Pser10 were larger and partially overlapping with H3K4me3 (Fig. 7, insets). We also noted that in S. papillosus H3Pser10 stains strongly only in the proximal part of the band of small nuclei (Fig. 7, S. papillosus panel and inset). Even though we used roughly age-matched worms (adult females) for these analyses, it must be noted that we did find indications in S. papillosus that staining patterns with H3Pser10 depended at least partially on age (Suppl Fig. 3B).

\section{Sex-specific histone modifications in Strongyloididae}

In parallel, we investigated sex-specific histone modification differences within the Strongyloididae, by analyzing male gonads in S. ratti, S. papillosus, and P. trichosuri with H3Pser10 and H3K4me3 (Fig. 8a, Table 2). We found that the staining patterns in the distal gonads were indistinguishable between males and females of the same species (Fig. 6 and Suppl Fig. 2C). However, the small nuclei in males are far more condensed than in free-living females, allowing clearer visualization of H3Pser10 and H3K4me3 localization within the nucleus (Fig. 8a, insets). In S. ratti male small nuclei, the H3Pser10 signal occupied nearly a third of the nucleus and was distinct from $\mathrm{H} 3 \mathrm{~K} 4 \mathrm{me} 3$, which occupied the remaining portion (see Fig. 8a, S. ratti inset). In $S$. papillosus males,
H3Pser10 stained the entire nucleus evenly, with this staining being much stronger in the proximal part of the band of small nuclei (Fig. 8a, S. papillosus inset), just like our observation in $S$. papillosus females. In P. trichosuri, condensed spermatogenetic chromosomes proximal to the small nuclei stained strongly for H3Pser10, which was never observed in either of the two species of Strongyloides (Fig. 8a, $P$. trichosuri inset). Interestingly, in parasitic $S$. ratti and S. papillosus females, we found $\mathrm{H} 3 \mathrm{~K} 4 \mathrm{me} 3$ and H3Pser10 staining patterns very similar to the ones in free-living males (Suppl Fig. 4). This is remarkable, given that parasitic females of Strongyloides are known to reproduce asexually by mitotic parthenogenesis and not undergo anything resembling meiosis.

\section{H3Pser10 is an X-chromosome-specific histone modification in $S$. ratti males}

In order to determine if the mutually exclusive regions staining for H3Pser10 and H3K4me3 represent different chromosomes, we performed FISH experiments with Xchromosome-specific and autosome-specific probes on dissected male $S$. ratti gonads (Fig. 8b). All our Xchromosome-specific FISH probes co-localized with the region of the nucleus displaying H3Pser10 staining (Fig 8b, top panel and inset). On the contrary, all autosome-specific probes did not co-localize with H3Pser10 staining (Fig 8b, bottom panel and inset). Therefore, at least in males, H3Pser10 appears to be an X-chromosome-specific histone modification in the small nuclei, indicating different chromatin modification states of the X-chromosome and the autosomes.

\section{Germline chromatin in $S$. ratti}

Finally, in order to gain a better understanding of germline chromatin, and in particular chromosome-specific histone modifications, we analyzed five other histone modifications in the S. ratti germline, in both sexes (Suppl Figs. 5 and 6, Table 3). The five-histone modifications selected are associated with either active transcription, e.g., H3K9/K14ac and H3K27ac, or chromatin silencing, e.g., H3K9me1, H3K27me3, and H4K20me1 (Kouzarides 2007; Schaner and Kelly 2006). For evidence of chromosomal-specificity, we always co-stained with one of the two modifications (H3K4me3 or H3Pser10) described above. Antibodies against H3K9/K14ac and H3K27ac (SupplFig. 5) stained both the giant and the small nuclei in both sexes strongly, potentially indicating active transcription. This is consistent with our H3K4me3 stainings. In addition, we found indications for sex-specific staining differences for both these markers. In females, these two histone modifications stained the different gonad compartments evenly, while in the males, certain regions stained more brightly than others. In particular, 


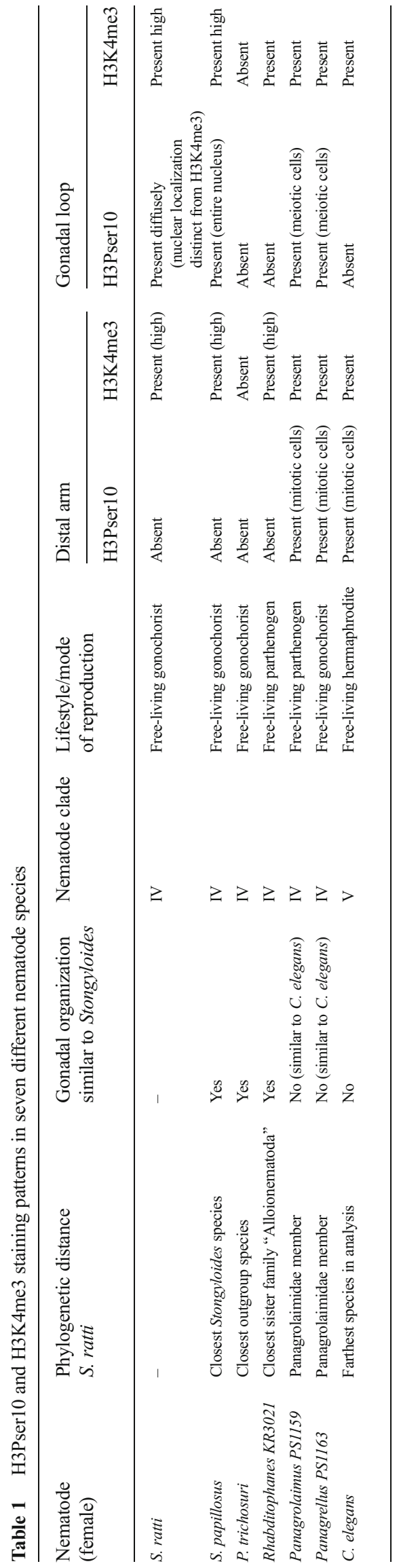

H3K27ac stained giant nuclei at the distal tip more intensely than over the gonad arm (Suppl Fig. 5, H3K27ac male and female distal panels). Furthermore, H3K27ac stained small nuclei in the distal part of the band in males more strongly than toward the proximal (Suppl Fig. 5, male and female gonadal loop panels), whereas H3K9/K14ac stained small nuclei at the proximal part of the band more intensely than toward the distal (Suppl Fig. 5, male and female gonadal loop panels).

Conversely, germlines of $S$. ratti stained against histone modifications known to be involved in silencing (H3K9me1, H3K27me3, and H4K20me1) showed only weak stainings (Suppl Fig. 6). In the giant nuclei of both males and females, H3K27me3 and H4K20me1 stainings could sometimes only be detected as bright puncta at the nuclear periphery, which is presumably stained heterochromatin (Suppl Fig. 6, distal gonad panels). Intriguingly, the only exception was H4K20me1, which stained the band region containing small nuclei in males very strongly (Suppl Fig. 6, male and female gonad loop panels). Overall, we observed similar staining patterns for all transcription activation markers used (namely H3K4me3, H3K9/K14ac, and H3K27ac), with all these modifications consistently staining both the giant and small nuclei. On the other hand, the silencing markers were absent or only weakly detected in these nuclei. The exact roles and functions of each of these histone modifications and their potential effect on germline development and regulation now need to be investigated further. Based on our results, H3Pser10 remains the only $\mathrm{X}$-chromosome-specific histone modification identified in the $S$. ratti male germline. Consequently, it appears that $S$. ratti not only demonstrates an altered germline structure but also altered chromatin regulation through differing histone modifications in comparison to C. elegans.

\section{Discussion}

Nematodes are powerful organisms in which to study the development and evolution of multiple organ systems. The genus Strongyloides consists of fairly close relatives displaying a wide range of lifestyles, with many species documented as economically relevant parasites of plants and animals (Blaxter et al. 1998; Holterman et al. 2006). However, a comparative approach for studying the evolution of the reproductive systems in such species has been lacking, compared to the well-studied but nonparasitic clade $\mathrm{V}$ nematodes like C. elegans and P. pacificus (Sommer and Bumbarger 2012). This work is therefore a first step toward bridging this gap using members of the genus Strongyloides.

Although C. elegans and Strongyloides nematodes share superficial morphological similarity and were classified as close relatives in the pre-molecular age (Blaxter et al. 1998), 
A Male small nuclei panels



Fig. 8 a A comparison of $\mathrm{H} 3 \mathrm{~K} 4 \mathrm{me} 3$ and $\mathrm{H} 3 \mathrm{P}$ ser10 staining patterns in the gonadal loop (containing small nuclei) of $S$. ratti, S. papillosus, and P. trichosuri males. Insets (extreme right) are magnified views of the areas marked in white in the merge panels, showing co-localization patterns with these two antibodies in each species. Note that the H3Pser10 staining is localized to a part of the nucleus in $S$. ratti, is evenly distributed in $S$. papillosus and found only on condensed meiotic chromosomes in P. trichosuri. Scale bar $10 \mu \mathrm{m}$. b FISH in S. ratti males. Top: the band

they differ greatly in many respects. One major difference is that the entire distal gonad of Strongyloides spp. consists of a population of endoduplicated giant nuclei, whereas the same of small nuclei in adult $S$. ratti males (for orientation, distal arm is to the top) showing co-localization of H3Pser10 (in green) and the Xchromosomal FISH probe (in red) within these nuclei. Inset (extreme right) is a magnified view of the region marked in white in the merge panel, with a rough outline of the nuclei (based on DAPI) marked as ovals using dashed lines. Bottom: the small nuclei in males show no colocalization (inset, extreme right) of H3Pser10 (in green) with an autosomal FISH probe (in red) within these nuclei. Scale bar $15 \mu \mathrm{m}$

region in C. elegans contains both proliferating germline stem cells and early meiotic nuclei. We, in agreement with earlier investigators (Hammond and Robinson 1994), have proposed 


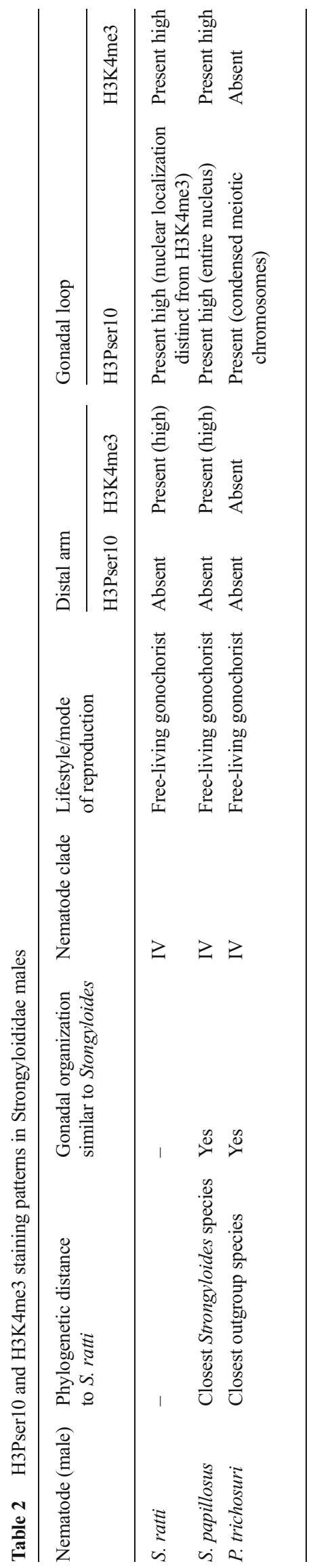

that the polyploid giant nuclei in these parasitic nematodes might have nurse cell activity. Given the short reproductive window of Strongyloides nematodes (Gardner et al. 2004, 2006), these nuclei possibly provide a rapid supply of material during oogenesis, potentially allowing germ cells to move quickly through meiosis and early embryonic development. In $C$. elegans, there appears to be no specialized population of cells fulfilling this sole function. Instead, meiotic cells in pachytene (which go on to give rise to oocytes) are thought to perform this role (Wolke et al. 2007). All our results presented here are consistent with this proposed nurse cell function. This includes a distal arm rich in ribosomes and mitochondria (indicative of an active protein machinery) along with consistently high immuno-stainings in the giant nuclei for markers associated with transcriptional activity. Quantitative DNA and RNA sequencing of the distal gonad provided further evidence for high gene expression in the giant nuclei (Kulkarni et al. 2015). This study showed that autosomal genes are predominantly expressed in these nuclei (in comparison to $\mathrm{X}$ chromosomal ones), which is (at least partially) achieved by differential DNA amplification.

A second striking difference compared to $C$. elegans appears to be the lack of proliferating germline stem cells in the adult Strongyloididae gonad. None of our observations (i.e., our detailed light and electron microscopic analyses or the BrdU incorporation experiments) yield any indication of active DNA replication in adults. Although we have consistently observed H3Pser10 staining in the small nuclei at the gonadal loop of Strongyloides members, we think that this staining is not indicative of nuclear divisions in these species. This is a major difference compared to other systems including C. elegans, where H3Pser10 staining is directly indicative of cells in M-phase, never staining cells in S-phase (Hsu et al. 2000; Kadyk and Kimble 1998). Instead H3Pser10, for example in the case of $S$. ratti, is restricted only to a part of the nucleus and presumably a modification specific to the $\mathrm{X}$-chromosome. Overall, we think that these observations together support the idea that Strongyloididae members build up a stock of germ cells during larval development, and then draw from this stock once they have matured. Indeed, free-living adults of these species are very short lived (one to two days of reproductive activity) and produce only a few dozen progeny (Gardner et al. 2004, 2006). For comparison, the longer-lived C. elegans can reproduce for several days and produce hundreds of progeny (Wegewitz et al. 2008).

Based upon its position at the distal tip, we have identified a somatic cell that may be the distal tip cell (DTC) in these species. The DTC in C. elegans has been implicated in maintaining a stem cell population in the distal gonad along with guiding the growth of the gonad arms during larval development (Kimble and Crittenden 2005). In this regard, it will be interesting to evaluate the developmental origin (lineage) of this DTC-like cell in Strongyloides spp., by performing 


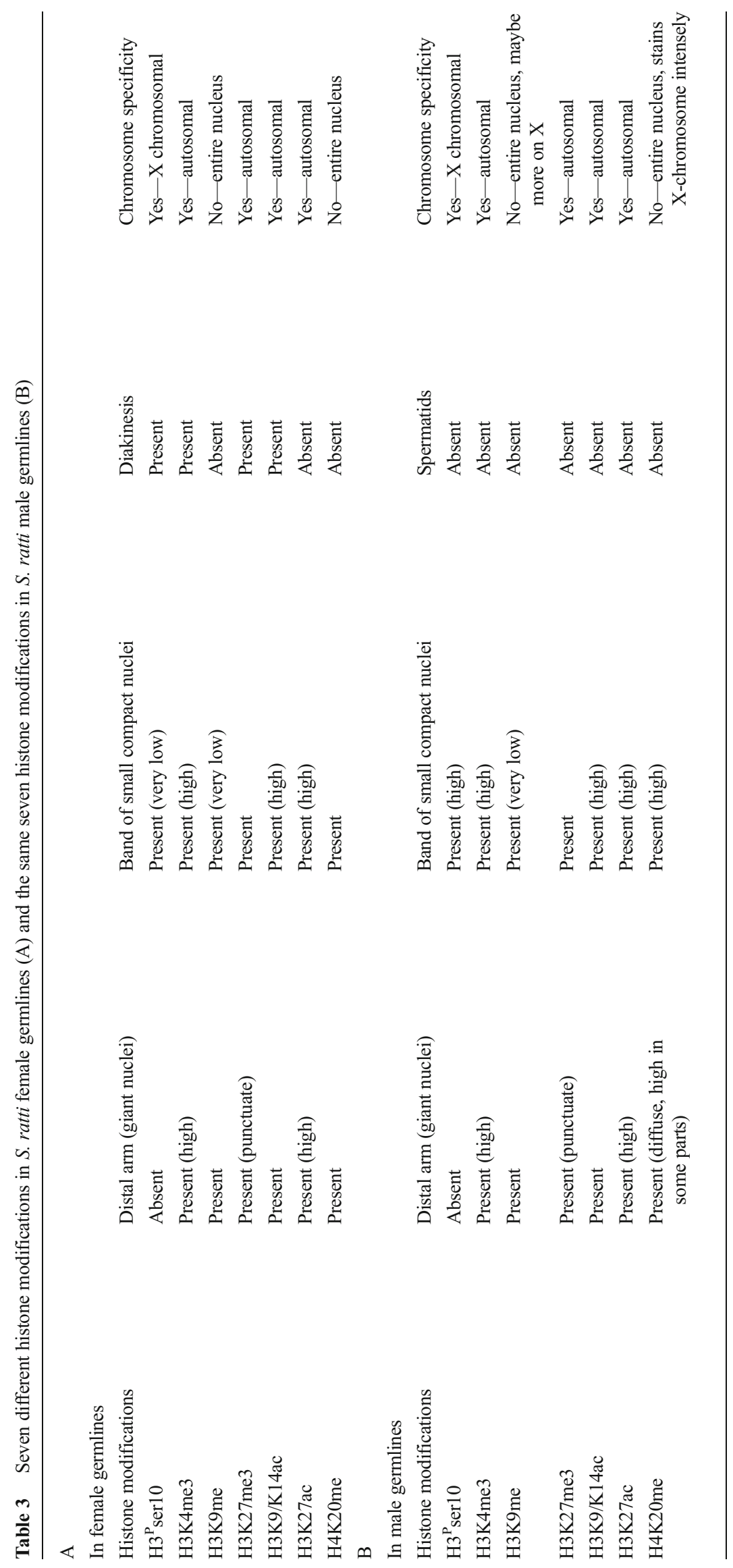


ablation experiments, and then studying its role in gonad development, organization, and function.

Until the recent discovery of DNA methylation on N6Adenine in C. elegans (Greer et al. 2015), it was thought that C. elegans lacks all DNA methylation (Simpson et al. 1986) and thus depends mainly on histone modifications to dictate chromatin structure. Histone modifications are thought to directly control function of local genomic regions, by increasing or decreasing the accessibility of DNA for transcription (Schaner and Kelly 2006). Since little is currently known about histone modifications in Strongyloides spp., to create an overview, we characterized multiple chromatin modifications of germ cells in S. ratti. We then focused on two modifications, namely H3K4me3 and H3Pser10 and used these for comparison with other clade IV nematodes and $C$. elegans.

In C. elegans, H3K4me3 brightly stains pachytene nuclei (in comparison to mitotic stem cells) known to be highly active transcriptionally (Schaner and Kelly 2006). The strong H3K4me3 stainings in the distal germlines (and at the distal tip) of Strongyloides and other clade IV nematodes (with the notable exception of $P$. trichosuri) might hint that the distal tip is a place of high transcriptional activity in these nematodes. The high presence of other transcriptional markers like H3K9/K14ac and H3K27ac at the distal tip (e.g., in S. ratti) provides further evidence for this. The absence of H3K4me3 in $P$. trichosuri is surprising and might possibly indicate that this modification is not a mark for active transcription in the giant nuclei of this species.

In the distal gonad of some clade IV nematodes (Panagrellus and Panagrolaimus) and C. elegans, H3Pser10 seems to be specific for dividing nuclei, implying that this role of H3Pser10 in the distal gonad may be conserved. In species without dividing cells in the distal gonad; we consistently did not observe any H3Pser10 staining in this part of the gonad. Surprisingly, in $S$. ratti males, H3Pser10 specifically marks a portion of the spermatogenic nuclei and based on the limited number of loci that we tested by FISH, this seems to be specific to the X-chromosome. Although some sperm without an X- chromosome (nullo-X sperm) exist in S. ratti (Kulkarni et al. 2015), it is likely that X-bearing sperm are preferentially made over nullo-X sperm, given that the males in this species sire only female progeny. Intriguingly, while H3Pser10 has been implicated in multiple gonad-related processes such as mitosis, apoptosis, and gametogenesis (Kouzarides 2007), it has also been implicated in dosage compensation (Wang et al. 2001), another process that requires distinguishing the $\mathrm{X}$-chromosome from the autosomes. In this regard, we found two other histone modifications, namely $\mathrm{H} 3 \mathrm{~K} 27 \mathrm{me} 3$ and H4K20me1 that are known to play a role in dosage compensation in other animals (Heard and Disteche 2006; Leeb and Wutz 2010; Payer and Lee 2008), to be abundant in the small compact nuclei of $S$. ratti males. This clearly indicates that further research is required to elucidate the functional consequences of these findings in these nematodes.

Interestingly, in S. papillosus, males lack an independent $\mathrm{X}$-chromosome, and H3Pser10 was found to stain the entire nucleus evenly. For $P$. trichosuri (a species with an independent $\mathrm{X}$-chromosome and known for producing male progeny), H3Pser10 marks both the condensed meiotic Xchromosomes and the autosomes alike. We never noted this modification on condensed meiotic chromosomes in either $S$. ratti or $S$. papillosus males. These striking and varied differences in $\mathrm{H} 3 \mathrm{~K} 4 \mathrm{me} 3$ and H3Pser10 stainings within the three closely related representatives of the Strongyloididae suggests that this group of nematodes has either recently undergone, or is still in the process of undergoing rapid evolutionary changes. This in turn may be a response to becoming parasitic and/or parthenogenetic (in the parasitic generation). Overall, due to the current limitation in knowledge and tools for Strongyloides species, we must assume that the differences between Strongyloides spp. and $C$. elegans with regard to the different histone modifications are probably due to a combination of stochastic changes caused either by drift or because of adaptations that have accumulated over the time of their phylogenetic separation (Fig. 9).
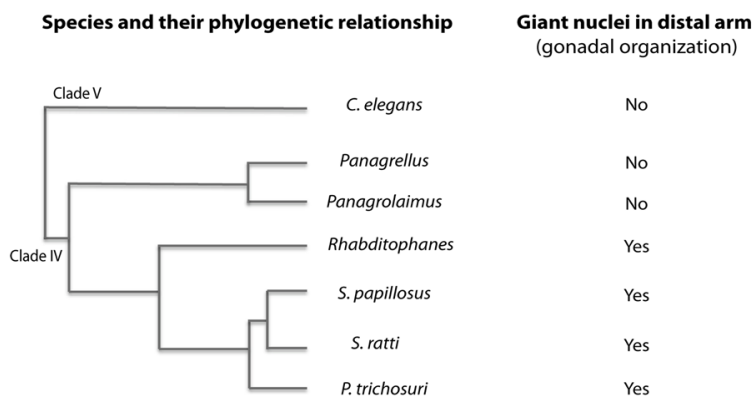

Fig. 9 Phylogenetic relationships, gonadal organization, lifestyles, and histone modifications in the nematode species studied. Species with a gonad organization similar to Strongyloides (Parastrongyloides and
Life style / Mode of reproduction

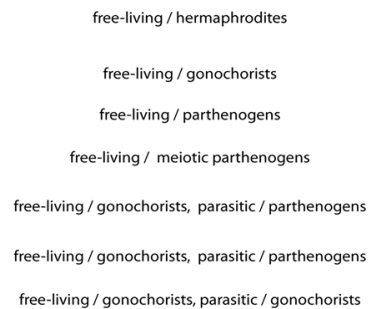

H3Pser10/ H3K4me3 staining

mitotic cells at distal tip (DT)/ throughout

mitotic (DT) and meiotic cells/ throughout

mitotic (DT) and meiotic cells/ throughout

no staining/throughout

presumably meiotic cells (entire nucleus)/ throughout

presumably meiotic cells (part of nucleus, Xchr)/ throughout (autosomal)

on condensed meiotic chromosomes/no staining
Rhabditophanes) lack H3Pser10 staining in their distal arms, while species (Panagrellus and Panagrolaimus) with a gonad organization similar to C. elegans show H3Pser10 in this region 
It must be noted that the Strongyloididae gonad is probably a highly derived structure, and therefore, care must be taken while drawing direct conclusions based on other systems. First, one has to keep in mind that the gonads of clade IV nematodes are not as well characterized as in C. elegans, and so, it is not easy to define corresponding populations of cells. Another issue with Strongyloides spp. is that they need to be grown in fecal cultures, where the development of individual worms cannot be easily followed (the age of individual worms can therefore only be estimated). Nevertheless, from our comparative observations of the staining patterns with C. elegans, it appears likely that the small compact nuclei at the gonadal loop in Strongyloides spp. represent nuclei in some stage of meiotic development. If this is true then this small region is equivalent to the extended meiotic zone in C. elegans (occupying nearly two thirds of the distal arm length). This conclusion is supported by two observations: firstly, there is no other region in the gonad of these species which could be meiotic (given that this region is preceded by highly endoduplicated nuclei, and followed directly by oocytes or sperm) and secondly, the Strongyloides species we studied are known for their normal diploid genetics (Eberhardt et al. 2007; Nemetschke et al. 2010b; Viney et al. 1993), meaning that haploid gametes must necessarily be formed. It is of immense interest cytologically that the small nuclei look very different from meiotic cells of $C$. elegans. In particular, no condensed chromosomes reminiscent of the ones in the different meiotic zones from C. elegans appear in this region in members of Strongyloididae. Strikingly, these nuclei also transition from having noncondensed chromosomes to fully condensed bivalents rather abruptly, without any nuclei in intermediate stages of chromosomal condensation.

This study has opened up many questions of basic biological interest. In particular, we do not know if the different types of nuclei (the giant and the small nuclei) that we have described transform into one another over reproductive life. Due to current technological limitations with working on these species, all our observations so far have been limited to fixed specimens (this work and Kulkarni et al. 2015). Hopefully in the future, we will be able to observe the dynamics of these processes using marked chromatin, for example by visualization of GFP-tagged histone proteins.

Our findings presented here enhance our understanding of the biology of a group of fascinating but poorly understood parasites. Additionally, they also illustrate the usefulness of the Rhabditophanes spp. (free-living)/ Parastrongyloides spp. (facultative parasite)/ Strongyloides spp. (obligate parasite with a single freeliving generation) in comparative studies. Given their multiple advantages, such systems could be used in the future to address questions about the emergence of parasitic lifestyles and the evolutionary consequences of becoming parasitic. Finally, novel insights into germline regulation during development may only be uncovered by looking into atypical species.

Acknowledgments Open access funding provided by Max Planck Society. We thank Anja Holz for help with gonad dissections, Dorothee Harbecke for technical support, Dee Denver for Rhabditophanes sp., Ralf J. Sommer for Panagrolaimus and Panagrellus sp., Metta Riebesell for TEM images of P. pacificus, and Alex Dulovic for proof reading the manuscript.

\section{Compliance with ethical standards}

Conflict of interest The authors declare that they have no conflict of interest.

Animal experiments All applicable international, national, and/or institutional guidelines for the care and use of animals were followed.

This article does not contain any studies with human participants performed by any of the authors.

Open Access This article is distributed under the terms of the Creative Commons Attribution 4.0 International License (http:// creativecommons.org/licenses/by/4.0/), which permits unrestricted use, distribution, and reproduction in any medium, provided you give appropriate credit to the original author(s) and the source, provide a link to the Creative Commons license, and indicate if changes were made.

\section{References}

Albertson DG, Nwaorgu OC, Sulston JE (1979) Chromatin diminution and a chromosomal mechanism of sexual differentiation in Strongyloides papillosus. Chromosoma 75:75-87

Basir MA (1950) The morphology and development of the sheep nematode, Strongyloides papillosus (Wedl, 1856). Can J Res 173-196

Blaxter ML, De Ley P, Garey JR, Liu LX, Scheldeman P, Vierstraete A, Vanfleteren JR, Mackey LY, Dorris M, Frisse LM, Vida JT, Thomas WK (1998) A molecular evolutionary framework for the phylum Nematoda. Nature 392:71-75

Bleay C, Wilkes CP, Paterson S, Viney ME (2007) Density-dependent immune responses against the gastrointestinal nematode Strongyloides ratti. Int J Parasitol 37:1501-1509

Chan RC, Chan A, Jeon M, Wu TF, Pasqualone D, Rougvie AE, Meyer BJ (2003) Chromosome cohesion is regulated by a clock gene paralogue TIM-1. Nature 423:1002-1009

Crittenden SL, Leonhard KA, Byrd DT, Kimble J (2006) Cellular analyses of the mitotic region in the Caenorhabditis elegans adult germ line. Mol Biol Cell 17:3051-3061

Crook M, Viney ME (2005) The effect of non-immune stresses on the development of Strongyloides ratti. Parasitology 131:383-392

Dorris M, Viney ME, Blaxter ML (2002) Molecular phylogenetic analysis of the genus Strongyloides and related nematodes. Int J Parasitol 32:1507-1517

Eberhardt AG, Mayer WE, Streit A (2007) The free-living generation of the nematode Strongyloides papillosus undergoes sexual reproduction. Int J Parasitol 37:989-1000

Fenton A, Paterson S, Viney ME, Gardner MP (2004) Determining the optimal developmental route of Strongyloides ratti: an evolutionarily stable strategy approach. Evol Int J Org Evol 58:989-1000

Gardner MP, Gems D, Viney ME (2004) Aging in a very short-lived nematode. Exp Gerontol 39:1267-1276 
Gardner MP, Gems D, Viney ME (2006) Extraordinary plasticity in aging in Strongyloides ratti implies a gene-regulatory mechanism of lifespan evolution. Aging Cell 5:315-323

Gemmill AW, Viney ME, Read AF (2000) The evolutionary ecology of host-specificity: experimental studies with Strongyloides ratti. Parasitology 120(Pt 4):429-437

Grant WN, Skinner SJ, Howes JN, Grant K, Shuttleworth G, Heath DD, Shoemaker CB (2006a) Heritable transgenesis of Parastrongyloides trichosuri: a nematode parasite of mammals. Int J Parasitol 36:475-483

Grant WN, Stasiuk S, Newton-Howes J, Ralston M, Bisset SA, Heath DD, Shoemaker CB (2006b) Parastrongyloides trichosuri, a nematode parasite of mammals that is uniquely suited to genetic analysis. Int J Parasitol 36:453-466

Greer EL, Blanco MA, Gu L, Sendinc E, Liu J, Aristizabal-Corrales D, Hsu CH, Aravind L, He C, Shi Y (2015) DNA methylation on N6adenine in C. elegans. Cell 161:868-878

Hammond MP, Robinson RD (1994) Endoreplication in the ovary, testis, and intestine of Strongyloides stercoralis. J Parasitol 80:905-910

Harvey SC, Viney ME (2001) Sex determination in the parasitic nematode Strongyloides ratti. Genetics 158:1527-1533

Heard E, Disteche CM (2006) Dosage compensation in mammals: finetuning the expression of the X chromosome. Genes Dev 20: $1848-1867$

Holterman M, van der Wurff A, van den Elsen S, van Megen H, Bongers T, Holovachov O, Bakker J, Helder J (2006) Phylum-wide analysis of SSU rDNA reveals deep phylogenetic relationships among nematodes and accelerated evolution toward crown clades. Mol Biol Evol 23:1792-1800

Hsu JY, Sun ZW, Li X, Reuben M, Tatchell K, Bishop DK, Grushcow JM, Brame CJ, Caldwell JA, Hunt DF, Lin R, Smith MM, Allis CD (2000) Mitotic phosphorylation of histone H3 is governed by Ipl1/ aurora kinase and Glc7/PP1 phosphatase in budding yeast and nematodes. Cell 102:279-291

Hubbard EJ, Greenstein D (2005) Introduction to the germ line (September 1, 2005), WormBook, ed. The C. elegans Research Community, WormBook, doi:10.1895/wormbook.1.18.1, http:// www.wormbook.org

Kadyk LC, Kimble J (1998) Genetic regulation of entry into meiosis in Caenorhabditis elegans. Development 125:1803-1813

Kimble J, Crittenden SL (2005) Germline proliferation and its control. WormBook: the online review of C. elegans biology 1-14

Kouzarides T (2007) Chromatin modifications and their function. Cell 128:693-705

Kulkarni A, Dyka A, Nemetschke L, Grant WN, Streit A (2013) Parastrongyloides trichosuri suggests that $\mathrm{XX} / \mathrm{XO}$ sex determination is ancestral in Strongyloididae (Nematoda). Parasitology 140: $1822-1830$

Kulkarni A, Holz A, Rödelsperger C, Harbecke D, Streit A (2015) Differential chromatin amplification and chromosome complements in the germline of Strongyloididae (Nematoda). Chromosoma. doi: 10.1007/s00412-015-0532-y

Leeb M, Wutz A (2010) Mechanistic concepts in X inactivation underlying dosage compensation in mammals. Heredity 105:64-70

Mackerras MJ (1959) Strongyloides and Parastrongyloides (Nematoda: Rhabdiasoidea) in Australian marsupials. Aust J Zool 7:87-104

Nemetschke L, Eberhardt AG, Hertzberg H, Streit A (2010a) Genetics, chromatin diminution, and sex chromosome evolution in the parasitic nematode genus Strongyloides. Curr Biol 20:1687-1696

Nemetschke L, Eberhardt AG, Viney ME, Streit A (2010b) A genetic map of the animal-parasitic nematode Strongyloides ratti. Mol Biochem Parasitol 169:124-127

Nigon V, Roman E (1952) Le déterminisme du sexe et le development cyclique de Strongyloides ratti. Bull Biol Fr Belg 86:404-448

Pasierbek P, Fodermayr M, Jantsch V, Jantsch M, Schweizer D, Loidl J (2003) The Caenorhabditis elegans SCC-3 homologue is required for meiotic synapsis and for proper chromosome disjunction in mitosis and meiosis. Exp Cell Res 289:245-255

Payer B, Lee JT (2008) X chromosome dosage compensation: how mammals keep the balance. Annu Rev Genet 42:733-772

Rinaldo C, Bazzicalupo P, Ederle S, Hilliard M, La Volpe A (2002) Roles for Caenorhabditis elegans rad-51 in meiosis and in resistance to ionizing radiation during development. Genetics 160: $471-479$

Rando OJ (2012) Combinatorial complexity in chromatin structure and function: revisiting the histone code. Curr Opin Genet Dev $22: 148-155$

Rudel D, Sommer RJ (2003) The evolution of developmental mechanisms. Dev Biol 264:15-37

Rudel D, Riebesell M, Sommer RJ (2005) Gonadogenesis in Pristionchus pacificus and organ evolution: development, adult morphology and cell-cell interactions in the hermaphrodite gonad. Dev Biol 277: 200-221

Schaner CE, Kelly WG (2006) Germline chromatin (January 24, 2006). In: Community, T.C.e.R. (Ed.), WormBook WormBook. doi:10. 1895/wormbook.1.73.1, http://www.wormbook.org

Shao H, Li X, Nolan TJ, Massey HC Jr, Pearce EJ, Lok JB (2012) Transposon-mediated chromosomal integration of transgenes in the parasitic nematode Strongyloides ratti and establishment of stable transgenic lines. PLoS Pathog 8:e1002871

Simpson VJ, Johnson TE, Hammen RF (1986) Caenorhabditis elegans DNA does not contain 5-methylcytosine at any time during development or aging. Nucleic Acids Res 14:6711-6719

Sommer RJ, Bumbarger DJ (2012) Nematode model systems in evolution and development. Wiley interdisciplinary reviews. Dev Biol $1: 389-400$

Stiernagle T (1999) Maintenance of $C$. elegans. In: Hope IA (ed) $C$. elegans a practical approach. Oxford University Press, Oxford, pp $51-67$

Streit A (2008) Reproduction in Strongyloides (Nematoda): a life between sex and parthenogenesis. Parasitology 135:285-294

Streit A (2014) How to become a parasite without sex chromosomes: a hypothesis for the evolution of Strongyloides spp. and related nematodes. Parasitology 141:1244-1254

Triantaphyllou AC, Moncol DJ (1977) Cytology, reproduction, and sex determination of Strongyloides ransomi and S. papillosus. J Parasitol 63:961-973

Viney ME (1999) Exploiting the life cycle of Strongyloides ratti. Parasitol Today 15:231-235

Viney ME, Lok JB (2015) The biology of Strongyloides spp. (July 16, 2015) WormBook, ed. The C. elegans Research Community, WormBook. doi:10.1895/wormbook.1.141.2, http://www. wormbook.org

Viney ME, Matthews BE, Walliker D (1992) On the biological and biochemical nature of cloned populations of Strongyloides ratti. J Helminthol 66:45-52

Viney ME, Matthews BE, Walliker D (1993) Mating in the nematode parasite Strongyloides ratti: proof of genetic exchange. Proc R Soc Biol Sci 254:213-219

Viney ME, Green LD, Brooks JA, Grant WN (2002) Chemical mutagenesis of the parasitic nematode Strongyloides ratti to isolate ivermectin resistant mutants. Int J Parasitol 32:1677-1682

Viney ME, Steer MD, Wilkes CP (2006) The reversibility of constraints on size and fecundity in the parasitic nematode Strongyloides ratti. Parasitology 133:477-483

Wang Y, Zhang W, Jin Y, Johansen J, Johansen KM (2001) The JIL-1 tandem kinase mediates histone $\mathrm{H} 3$ phosphorylation and is required for maintenance of chromatin structure in Drosophila. Cell 105: 433-443

Wegewitz V, Schulenburg H, Streit A (2008) Experimental insight into the proximate causes of male persistence variation among two 
strains of the androdioecious Caenorhabditis elegans (Nematoda). BMC Ecol 8:12

Wolke U, Jezuit EA, Priess JR (2007) Actin-dependent cytoplasmic streaming in C. elegans oogenesis. Development 134:2227-2236
Yamada M, Matsuda S, Nakazawa M, Arizono N (1991) Species-specific differences in heterogonic development of serially transferred freeliving generations of Strongyloides planiceps and Strongyloides stercoralis. J Parasitol 77:592-594 